\title{
Magnetic field-aligned plasma currents in gravitational fields
}

\author{
O. E. Garcia ${ }^{1}$, E. Leer ${ }^{2}$, H. L. Pécseli ${ }^{3}$, and J. K. Trulsen ${ }^{2}$ \\ ${ }^{1}$ Department of Physics and Technology, UiT - The Arctic University of Norway, 9037 Troms $\varnothing$, Norway \\ ${ }^{2}$ Department of Theoretical Astrophysics, University of Oslo, 0315 Oslo, Norway \\ ${ }^{3}$ Department of Physics, University of Oslo, 0316 Oslo, Norway
}

Correspondence to: H. L. Pécseli (hans.pecseli@fys.uio.no)

Received: 3 December 2014 - Revised: 28 January 2015 - Accepted: 30 January 2015 - Published: 3 March 2015

\begin{abstract}
Analytical models are presented for currents along vertical magnetic field lines due to slow bulk electron motion in plasmas subject to a gravitational force. It is demonstrated that a general feature of this problem is a singularity in the plasma pressure force that develops at some finite altitude when a plasma that is initially in static equilibrium is set into slow motion. Classical fluid models thus do not allow general steady-state solutions for field-aligned currents. General solutions have to be non-stationary, varying on time scales of many periods of a plasma equivalent to the BruntVäisälä frequency. Except for very special choices of parameters, a steady-state solution exists only in an average sense. The conditions at large altitudes turn out to be extremely sensitive to even small changes in parameters at low altitudes. Low frequency fluctuations detected at large altitudes in the polar regions need not be caused by local low frequency instabilities, but merely reflect small fluctuations in conditions at low altitudes.
\end{abstract}

Keywords. Ionosphere (electric fields and currents)

\section{Introduction}

Plasma atmospheres in magnetic polar regions have a density gradient along magnetic field lines. Steady-state static solutions are readily obtained for these cases where we have a balance between gravitational effects and thermal particle pressures (Pannekoek, 1922; Rosseland, 1924). In general both ions and electrons will contribute, although we note that for most relevant ionospheric cases the electron temperature is larger than the ion temperature, $T_{\mathrm{e}}>T_{\mathrm{i}}$. These steady-state conditions are often disturbed by magnetic field-aligned currents.
The simplest equilibrium solution is found for the isothermal case $T_{\mathrm{e}}=T_{\mathrm{i}} \equiv T$, with the plasma density varying as $n=n_{0} \exp \left(-\frac{1}{2} z(m+M) g / T\right)$. In this case the constant gravitational force $n(m+M) g$ balances the plasma pressure force $2 T \mathrm{~d} n / \mathrm{d} z$. It might be assumed that given a static exponential density variation without field-aligned currents (de la Beaujardiere et al., 1993), we also have this variation with fieldaligned currents, and then use this exponential form for calculating an integrated resistivity, etc. The argument is, however, misleading. As soon as the electrons flow along the vertical magnetic field lines (or nearly vertical: the magnetic field only needs to have a significant vertical component) there is no longer a simple altitude-density relation. Even a small electron flux introduced at the lowest part of an initially exponential vertical density profile can give rise to large relative variations at higher altitudes because of the exponentially small plasma density there, ultimately resulting in a pressure discontinuity. Since $n \rightarrow 0$ for $z \rightarrow \infty$, even infinitesimal perturbations propagating upwards from a given altitude will lead to relative perturbations that become large at some altitude.

The present paper addresses the conditions with electrons flowing along magnetic field lines, emphasising the case of field-aligned currents. The analysis will be restrictive in the sense that only bulk electron flows will be considered, thus excluding cases where an electron beam is causing the current. We argue, by giving an example for a simple yet realistic generator model, that such bulk motions are particularly relevant for the polar ionospheres. The generator model has interest in itself, but serves here also to allow an estimate of the magnitude of electron flow velocities that can be expected.

The paper is organised as follows: in Sect. 2 we present a simple model for a likely source of field-aligned bulk electron flow in the ionospheric $\mathrm{E}$ and $\mathrm{F}$ regions, where the free 
energy for the generator mechanism originates from a steadystate electric field perpendicular to the magnetic field lines. Some preliminary results of this model generator have been published before (Garcia and Pécseli, 2013). In Sect. 3 we discuss a simplified analytical model with cold ions that illustrates the basic problem associated with currents along magnetic field lines in plasmas with vertical density gradients. In particular the model serves to illustrate that Lambert's W-functions enters naturally in solutions for problems of this type, a detail that could otherwise be "lost" if the full set of equations were addressed from the outset. The model of Sect. 3 is appealing, but contains some simplifications which makes it unrealistic (albeit not nonphysical). Several of these simplifying assumptions are remedied in Sect. 4. Finally, Sect. 5 contains our conclusions.

\section{Battery mechanism}

The basic problem approached in the present study concerns bulk electron flows in plasmas in gravitational fields. This problem will be particularly relevant for plasma motions in polar regions where the magnetic field lines have a dominant vertical component. In this section we first discuss a simple model for a generator that induces magnetic fieldaligned electron flows. Currents can be caused by diffuse auroral electron precipitation that is generally distributed over a large spatial region (Ossakow and Chaturvedi, 1979). Here we focus on a different current generating mechanism, induced by steady-state horizontal electric fields $\boldsymbol{E}_{0}$ imposed perpendicular to $\boldsymbol{B}$ in the ionospheric $\mathrm{E}$ region. The basic generator mechanism is due to differences of electron and ion mobilities in the E region where $\omega_{\mathrm{ce}} \gg v_{\mathrm{en}}$ while $\Omega_{\mathrm{ci}} \leq v_{\mathrm{in}}$. The electron and ion collision frequencies with the neutral background gas are $v_{\mathrm{en}}$ and $v_{\text {in }}$. These neutral collision frequencies decrease rapidly with altitude (Gurevich, 1978; Kelley, 1989; Dyrud et al., 2006), see for instance Fig. 1. For altitudes above some $120-130 \mathrm{~km}$, the electrons and ions are both drifting with approximately the same $\boldsymbol{E}_{0} \times \boldsymbol{B} / B^{2}$ velocity. We model the $\mathrm{E}$ region as a collisional horizontal "slab" of thickness $D$ with $\omega_{\text {ce }} \gg v_{\text {en }}$ while $\Omega_{\text {ci }} \leq v_{\text {in }}$. For altitudes above approximately $120 \mathrm{~km}$, we ignore collisions altogether, see Fig. 1. The mobilities for single particle motion in the Hall current direction are given through

$\mathbf{U}_{\mathrm{H}}=\frac{1}{1+v^{2} / \omega_{\mathrm{c}}^{2}} \frac{\boldsymbol{E}_{0} \times \boldsymbol{B}}{B^{2}}$,

where $\omega_{\mathrm{c}}$ and $v$ are the cyclotron and neutral collision frequencies for the respective species, and $\mathbf{U}_{\mathrm{H}}$ is the appropriate velocity component in the Hall current direction. Applying this expression to the electron velocity within the given limiting parameter values we find $U_{\mathrm{e}} \approx E_{0} / B$ and $U_{\mathrm{i}} \approx\left(\Omega_{\mathrm{ci}} / \nu_{\mathrm{en}}\right)^{2} E_{0} / B \ll U_{\mathrm{e}}$. We will ignore the steady-state ion velocity in comparison to the electron velocity so that the relative velocity becomes $V_{\mathrm{d}} \equiv U_{\mathrm{e}}-U_{\mathrm{i}} \approx U_{\mathrm{e}}$ in the E re-

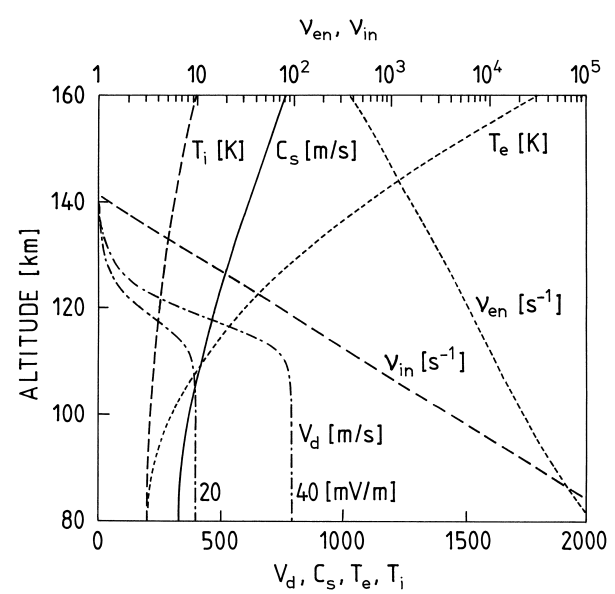

Figure 1. Altitude variation of collision frequencies, $v_{\mathrm{e}}, v_{\mathrm{i}}$, and temperatures $T_{\mathrm{e}}, T_{\mathrm{i}}$, together with the corresponding variation of the sound speed, with $V_{d}$ being the difference between the ion and electron drift velocities calculated for $E_{0}=20$ and $40 \mathrm{mV} \mathrm{m}^{-1}$. Note that the collisions frequencies have variable values at the top, while parameters like $C_{\mathrm{s}}, T_{\mathrm{e}}, T_{\mathrm{i}}, V_{\mathrm{d}} \equiv U_{\mathrm{e}}-U_{\mathrm{i}}$ have their values on the bottom axis. For the altitude range shown we can take the electron and ion cyclotron frequencies to be constant, $\omega_{\mathrm{ce}}=9.5 \times 10^{6}$ and $\Omega_{\mathrm{ci}} \approx 170 \mathrm{rad} \mathrm{s}^{-1}$, respectively, with $\Omega_{\mathrm{ci}}$ corresponding to an average ion mass of $31 \mathrm{amu}$.

gion, while $V_{\mathrm{d}} \approx 0$ in the $\mathrm{F}$ region. As far as the steadystate electron-ion velocity differences are concerned, this is a good approximation.

We have a net Hall current (the electrojet current) due to the neutral drag on the ions (Stubbe, 1968) that implies that electron and ion steady state drifts differ (Primdahl and Spangslev, 1977). The model for the current generation can also be understood in the $\boldsymbol{E}_{0} \times \boldsymbol{B}$-moving frame, where the neutral component is in motion. To interpret the free energy driving the current as an externally imposed electric field $\boldsymbol{E}_{0}$ or a neutral wind is merely a question of choosing the frame of reference.

If we now have a local plasma density enhancement with a density gradient perpendicular to $\boldsymbol{B}$ with $\nabla_{\perp} n_{0} \| \boldsymbol{E}_{0} \times \boldsymbol{B}$ as in Fig. 2, we have a local enhancement of the net current in that region since $\boldsymbol{E}_{0}$ is imposed externally to give a constant velocity while the number of charge carriers is locally enhanced. The height integrated electron current per unit length in the direction perpendicular to $\nabla_{\perp} n_{0}$ is denoted $I_{0}+I_{1}$ in the enhanced density region (with density $n_{0}+\Delta n$ between the two intervals $a$ and $b$ in Fig. 2). This current is not compensated at the boundaries of this magnetic flux tube: in regions $a$ and $b$, the current $I_{1}$ therefore has to expand along the vertical magnetic field lines as illustrated in Fig. 2. It can not escape downward into the D region because of the high collisionality there with a corresponding low electron mobility. Upwards, into the F region, we can have a current propagating (Primdahl and Spangslev, 1977; Primdahl et al., 1987). 


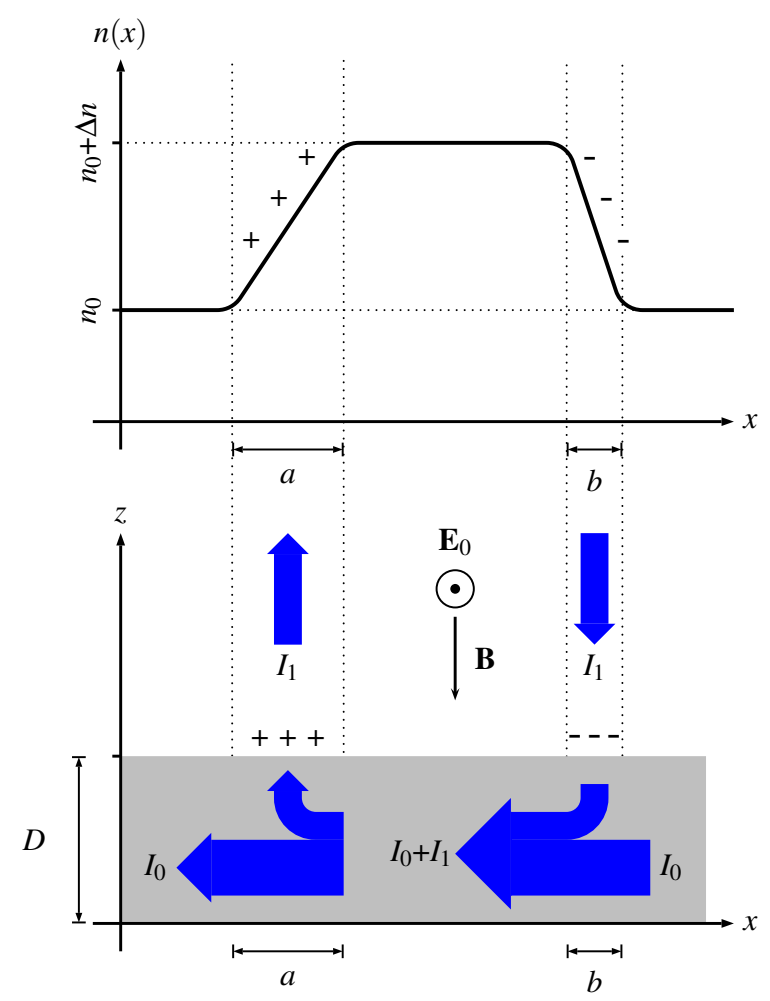

Figure 2. Illustration of a vertical cross-section through a magnetic flux tube with enhanced plasma density. At the top we show details of the geometry and density variations, while at the bottom of the figure we illustrate the currents generated by a steady-state electric field $\boldsymbol{E}_{0} \perp \boldsymbol{B}$. The thickness of the E region is $D \approx 10-20 \mathrm{~km}$.

This current will flow along magnetic field lines and in the direction perpendicular to $\nabla_{\perp} n_{0}$.

The present simple battery model has relations to standard models for auroral arcs (Boström, 1964; Prölss, 2004). The density enhancement model ensures that a current will always have a return current (see Fig. 2) so there is no net charge building up. The present model corresponds to a current generator and has no a priori imposed potential drop.

The current $I_{1}$ in the model illustrated in Fig. 2 is carried by the electrons, both in the ionospheric $\mathrm{E}$ and $\mathrm{F}$ regions. Since the DC ion mobility is low in the E region, we can assume the ion drift velocity there to be negligible. By the ion continuity equation, this assumption has the consequence that the bulk ion velocity is also negligible at high altitudes. Due to the abundance of electrons in the E region, a quasi steady state is achieved rapidly where electrons are flowing from the large plasma density $\mathrm{E}$ region into the smaller density in the $\mathrm{F}$ region. On the side facing the $\boldsymbol{E}_{0} \times \boldsymbol{B}$-drift in region $a$ in Fig. 2, the electrons have to flow from a smaller plasma density into the larger density in the $\mathrm{E}$ region. In this latter case, stationary conditions will need more time to become established. In region $b$ in Fig. 2 the electron drift will enhance upward travelling low frequency waves (Garcia and
Pécseli, 2013), in region $a$ unstable waves are propagating in the opposite direction.

The conservation of net current $\left(I_{0}+I_{1}\right)$ through the crosssection between regions $a$ and $b$ in Fig. 2 will act as an amplification for current densities and average electron flow velocities. For regions outside the enhanced plasma density flux tube we have a net electrojet current obtained by integrating over the E region, i.e. $I_{0}=J_{0} D=e n_{0} D E_{0} / B$. By Kirchhoff's laws we have, for instance, at the region $b$ in Fig. 2 where $J_{1}$ is the current density, that $e D \Delta n E_{0} / B \approx J_{1} b=$ $b e\left(n_{0}+\Delta n\right) U_{\mathrm{e}}$, giving the vertical electron drift velocity estimate $U_{\mathrm{e}} \approx\left(\Delta n /\left(n_{0}+\Delta n\right)\right)(D / b) E_{0} / B$. In the ionospheric E region (Kelley, 1989) we often have $E_{0} \geq 20 \mathrm{mV} \mathrm{m}^{-1}$, which gives $E_{0} / B \approx C_{\mathrm{s}}$, with $C_{\mathrm{s}}$ being the ion sound speed, so we can argue that substantial electron drifts can be achieved by this mechanism. In Fig. 1 we show two examples for relative ion-electron horizontal flow velocities induced by $\boldsymbol{E}_{0} \perp \boldsymbol{B}$ in the collisional ionospheric plasma, i.e. examples for $E_{0}=20$ and $40 \mathrm{mV} \mathrm{m}^{-1}$.

Magnetic field-aligned currents in the polar ionospheres are observed indirectly by the magnetic field variations they give rise to or measured "in situ" via instrumented spacecrafts (Smiddy et al., 1977; Primdahl et al., 1979; Lyons et al., 1979; Primdahl et al., 1987). Observed current densities have a large range of variation, $1-50 \mu \mathrm{A} \mathrm{m}^{-2}$ are reported, often observed as two current sheaths with opposite current directions. The variability of these currents along the space-craft trajectories are large, and it is often assumed that the lower limit of the thickness of current layers is determined by the electron Larmor radius, while an upper limit may be given by the size of the auroral oval. The variability of the current density is usually assumed to be due to a spatial variation that is sampled by the moving rocket, but with one rocket only the variation cannot be distinguished from a larger scale temporal variability.

The field-aligned currents can have sufficient intensity to make the plasma unstable (Kindel and Kennel, 1971; Garcia and Pécseli, 2013) and are important for the conditions of the ionospheric plasma. By plasma currents, here we mean bulk electron flows. Conditions where the currents are due, for instance, to energetic electron beams require a different analysis. We note, however, that in cases where field-aligned currents are due to fast electron beams, the return current is generally expected to be carried by slow bulk electron motions (Arnoldy, 1974).

For a numerical estimate we take a plasma density near the ionospheric $\mathrm{F}$ maximum of $10^{12}-10^{13} \mathrm{~m}^{-3}$ : in this case, a current density of $J \approx 50 \mu \mathrm{A} \mathrm{m}^{-2}$ corresponds to an electron velocity of $30 \mathrm{~m} \mathrm{~s}^{-1}$, i.e. $10-20 \%$ of the ion sound speed, $C_{\mathrm{s}}$. At higher altitudes, $1500 \mathrm{~km}$ for instance, the plasma density decreases to approximately $5 \times 10^{8} \mathrm{~m}^{-3}$, as observed by the Freja satellite (Khotyaintsev et al., 2001), and the electron flow velocity has to increase correspondingly in order to maintain a constant net vertical current. 


\section{Perturbations of a steady state: a simple model}

To illustrate the basic problem addressed in this work we now consider an analytically solvable steady-state model for the case where density gradients are aligned with magnetic field lines. To simplify the problem we let the magnetic field be vertical along the $z$ axis, with gravitational acceleration $g$ be in the negative $z$ direction, and consider a one-dimensional model. We have a momentum equation for isothermal electrons with density $n_{\mathrm{e}}$, bulk fluid velocity $u_{\mathrm{e}}$, charge $-e$, mass $m$ and temperature $T_{\mathrm{e}}$,

$n_{\mathrm{e}} m u_{\mathrm{e}} \frac{\mathrm{d} u_{\mathrm{e}}}{\mathrm{d} z}=-e n_{\mathrm{e}} E-\frac{\mathrm{d}\left(T_{\mathrm{e}} n_{\mathrm{e}}\right)}{\mathrm{d} z}-m n_{\mathrm{e}} g$,

and similarly a momentum equation for the ions with temperature $T_{\mathrm{i}}$, charge $e$ and mass $M$,

$n_{\mathrm{i}} M u_{\mathrm{i}} \frac{\mathrm{d} u_{\mathrm{i}}}{\mathrm{d} z}=e n_{\mathrm{i}} E-\frac{\mathrm{d}\left(T_{\mathrm{i}} n_{\mathrm{i}}\right)}{\mathrm{d} z}-M n_{\mathrm{i}} g$.

Considering the simple generator model outlined in Sect. 2 we assume that the current is carried by the electrons so that $u_{\mathrm{e}} \neq 0$ and take at first $u_{\mathrm{i}}=0$. This generator model injects slow upward moving electrons on one side of a magnetic field-aligned density enhancement. In the standard terminology of electrical circuits, this model corresponds to a current generator different from a voltage generator applied between the bottom and top of the ionosphere: we find the former to be most relevant here.

\section{Model problem with $T_{\mathrm{i}}=0$}

For the simplest case with $T_{\mathrm{i}} \approx 0$, we have $E=M g / e$. With a constant electric field the Poisson equation gives $n_{\mathrm{e}}=n_{\mathrm{i}}$. We thus do not have to assume charge neutrality or quasineutrality explicitly, it follows automatically here irrespective of the Debye length. If also $u_{\mathrm{e}}=u_{\mathrm{i}}=0$ we find a simple solution $n_{\mathrm{e}}=n_{0} \exp \left(-z(M+m) g / T_{\mathrm{e}}\right)$, where $n_{0}$ is the plasma density at $z=0$. In this equilibrium, the electron pressure sets up an electric field that balances the gravitational force on the ions. There is no current associated with this electric field.

For $u_{\mathrm{i}}=0, u_{\mathrm{e}} \neq 0$, the electron continuity equation with a constant outgoing electron flux gives $u_{\mathrm{e}} n_{\mathrm{e}}=u_{\mathrm{e} 0} n_{0}$. Since the plasma density decreases with increasing altitude, this implies that electrons must be accelerated in the positive $z$ direction.

With $E \equiv-\mathrm{d} \phi / \mathrm{d} z$, where $\phi$ is the electrostatic potential, we find the following result by integrating the electron momentum equation with constant $T_{\mathrm{e}}$ :

$$
\begin{aligned}
\frac{m}{2} \frac{\left(u_{\mathrm{e} 0} n_{0}\right)^{2}}{n^{2}} & =e \phi-T_{\mathrm{e}} \ln \left(\frac{n}{n_{0}}\right)+\frac{m}{2} u_{\mathrm{e} 0}^{2}-m g z \\
& =-M g z-T_{\mathrm{e}} \ln \left(\frac{n}{n_{0}}\right)+\frac{m}{2} u_{\mathrm{e} 0}^{2}-m g z .
\end{aligned}
$$

The choice of an integration constant ensures that the electrostatic potential $\phi=0$ at $z=0$, where $n=n_{0}$. For small $z$, the term on the left side of Eq. (4) is negligible due to the smallness of the electron inertia. However, as the density decreases for large $z$, the velocity increases, and eventually the left hand term becomes important. On the other hand, we can approximate $(m+M) g z \approx M g z$ in Eq. (4) and ignore the effect of the gravitational force on the electrons as compared to that on the ions. Our steady-state model here, and its generalisations in Sect. 4, differ from models discussed by Knight (1973) by retaining the gravitational effects that create the vertical density gradient, which in turn gives rise to a velocity variation through the continuity equation. As shown in the following, the resulting basic equations and their generalisations do not allow a global steady-state solution with a relative electron-ion flow.

In normalised units with $\eta \equiv n_{\mathrm{e}} / n_{0}$ and $\xi \equiv z / L$, with $L \equiv C_{\mathrm{s}}^{2} / g$, we have from Eq. (4):

$\xi-A^{2}=-\frac{A^{2}}{\eta^{2}}-\ln \eta$,

or

$2\left(\xi-A^{2}\right)=-\ln \left(\eta^{2} \exp \left(\frac{2 A^{2}}{\eta^{2}}\right)\right)$,

giving

$-2 A^{2} \exp \left(2\left(\xi-A^{2}\right)\right)=-\frac{2 A^{2}}{\eta^{2}} \exp \left(-\frac{2 A^{2}}{\eta^{2}}\right)$,

with $A^{2} \equiv \frac{1}{2}(m / M) u_{\mathrm{e} 0}^{2} / C_{\mathrm{s}}^{2} \geq 0$, where $C_{\mathrm{s}}=\sqrt{T_{\mathrm{e}} / M}$ is the ion sound speed. Only the magnitude of $u_{\mathrm{e} 0}$ enters $A$ in Eq. (6), not its direction. The expression (6) gives the normalised vertical position (i.e. altitude) as a function of plasma density, see also Fig. 3 that shows $\xi=\xi(\eta)$ as given by Eq. (5). The expression (6) already indicates the root of the problem. We expect that the plasma density varies monotonically with $\xi$, but see readily that the right hand side of Eq. (6) has a lower bound and varies monotonically in two intervals separated by the local maximum at $\eta=\sqrt{2 A^{2}}$. The altitude variable $\xi$ on the left side of Eq. (6) has, on the other hand, unbounded variation.

The expression (6) can be inverted analytically by introducing Lambert's $W$ function (or the "ProductLog" function) that often appears in physical problems (Valluri et al., $2000)$, sometimes unexpectedly. The function $W(x)$ returns the principal solution for $\zeta$ in $x=\zeta \exp (\zeta)$, where $W(x)$ is real for $x>-\exp (-1)$. In Fig. 4 we give some basic details, specifying in particular the two real branches $W_{0}$ and $W_{-1}$. For the normalised density, we find here that

$\eta^{2}(\xi)=-\frac{2 A^{2}}{W_{0}\left(-2 A^{2} \exp \left(-2 A^{2}+2 \xi\right)\right)}$.

With $\eta$ being a physical quantity, we have the left side of Eq. (8) being positive. Unphysical solutions have the right hand side of Eq. (8) being negative or complex, where we 


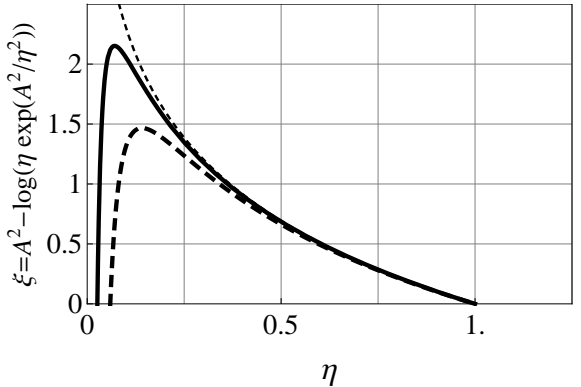

Figure 3. Variation of $\xi=A^{2}-\ln \left(\eta \exp \left(A^{2} / \eta^{2}\right)\right)$ with varying $\eta$, shown here for $A=0.05$ and 0.1 with full and dashed lines, respectively. The thin dashed line gives the gravitational exponential atmosphere without electron flow, i.e. $A=0$.

recall that Lambert's $W$ function can be complex. The normalised electron plasma pressure force $-\mathrm{d} \eta / \mathrm{d} \xi$ becomes singular at $\xi=\xi_{\mathrm{c}} \equiv A^{2}-\frac{1}{2}\left(1+\ln \left(2 A^{2}\right)\right)$, where $\xi_{\mathrm{c}} \approx-\ln |A|$ for small $|A|$. The electron density is no longer real-valued when $\xi>\xi_{\mathrm{c}}$. At $\xi=\xi_{\mathrm{c}}$ we find a singularity in plasma pressure while $u_{\mathrm{e}}=\sqrt{T_{\mathrm{e}} / m}$ there.

A special case is found for $A^{2}=1 / 2$, where $\xi_{\mathrm{c}}=0$. For this particular parameter choice we have $\eta^{2}(\xi)=$ $-1 / W_{0}(-\exp (2 \xi-1))$, but we note that by its definition, this $A$ value corresponds to $u_{\mathrm{e} 0}=C_{\mathrm{s}} \sqrt{M / m}$, which is a very large and unrealistic value.

The sensitivity of $\xi_{\mathrm{c}}$ to parameter variations is usually expressed by the derivative, here $\mathrm{d} \xi_{\mathrm{c}} / \mathrm{d} A=2 A-1 / A$, which is large when $A$ is small. The conditions at large altitudes are extremely sensitive to even small changes in parameters at low altitudes.

We can also express the normalised electron bulk flow velocity $\zeta \equiv u_{\mathrm{e}} / C_{\mathrm{s}}$ by first rewriting Eq. (2) as

$$
\begin{aligned}
\frac{m}{M} \frac{\mathrm{d} u_{\mathrm{e}}}{\mathrm{d} z} & =-e \frac{E}{M u_{\mathrm{e}}}-\frac{T_{\mathrm{e}}}{M} \frac{\mathrm{d}}{\mathrm{d} z} \frac{1}{u_{\mathrm{e}}} \\
& =-\frac{g}{u_{\mathrm{e}}}+\frac{C_{\mathrm{s}}^{2}}{u_{\mathrm{e}}^{2}} \frac{\mathrm{d} u_{\mathrm{e}}}{\mathrm{d} z},
\end{aligned}
$$

where we used $e E=M g$. With the normalised units used before, we would write this expression as

$$
\left(\frac{m}{M}-\frac{1}{\zeta^{2}}\right) \frac{\mathrm{d} \zeta^{2}}{\mathrm{~d} \xi}=-2
$$

Since the right hand side is always negative, and the electron velocity has to increase with altitude for a decreasing density, i.e. $\mathrm{d} \zeta^{2} / \mathrm{d} \xi>0$, we require $\zeta^{2}<M / m$, or in physical units $u_{\mathrm{e}}<\sqrt{T_{\mathrm{e}} / m}$. Since $|\mathrm{d} \zeta / \mathrm{d} \xi| \rightarrow \infty$ as $|\mathrm{d} \eta / \mathrm{d} \xi| \rightarrow \infty$ as $\xi \rightarrow$ $\xi_{\mathrm{c}}$, we must have $\zeta^{2} \rightarrow M / m$ as $\xi \rightarrow \xi_{\mathrm{c}}$, implying that the electron flow reaches the thermal electron velocity there.

The simplified fluid model outlined here does not allow a general steady state solution for a field-aligned current - the solution must be non-stationary. A characteristic

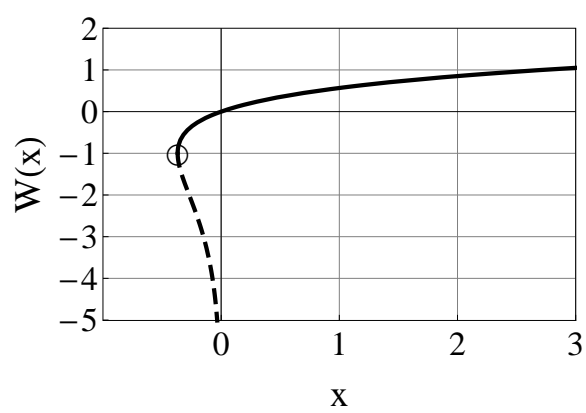

Figure 4. Illustration of the two real branches of Lambert's $W$ function. Full line gives $W_{0}(x)$, dashed line $W_{-1}(x)$. There is a branchcut at $x=-1 / \exp (1)$, marked by $\circ$.

reference frequency can be estimated by $\Omega_{\mathrm{BV}}=g / C_{\mathrm{s}}$. Using a definition of the Brunt-Väisälä frequency in the form $\sqrt{-g \mathrm{~d}\left(\ln n_{0}\right) / \mathrm{d} z}$ we note that $\Omega_{\mathrm{BV}}$ is a plasma equivalent to the Brunt-Väisälä frequency. With $C_{\mathrm{s}} \approx 500 \mathrm{~m} \mathrm{~s}^{-1}$, we have $\Omega_{\mathrm{BV}} \approx 2 \times 10^{-2} \mathrm{~s}^{-1}$ for the Earth's gravitational acceleration $g \approx 9.8 \mathrm{~m} \mathrm{~s}^{-2}$. A characteristic time scale $\tau_{\mathrm{c}}$ for the formation of the singularity associated with the $W$ function is then the length scale $\xi_{\mathrm{c}} L$ divided by a characteristic velocity, the sound speed $C_{\mathrm{s}}$, giving $\tau_{\mathrm{c}} \equiv \xi_{\mathrm{c}} L / C_{\mathrm{s}}=\xi_{\mathrm{c}} C_{\mathrm{s}} / g=\xi_{\mathrm{c}} / \Omega_{\mathrm{BV}} \gg$ $1 / \Omega_{\mathrm{BV}}$. The time scale is decreasing for increasing flow velocities. The system tries to build-up a modified steady state in response to an electron flow induced by a horizontal electric field $\boldsymbol{E}_{0}$, but since the state cannot be stationary it breaks down, and the process repeats, resulting in slow pulsations of the ionosphere.

\section{Generalisations of the model}

For the simple idealised model, we can conclude that the plasma cannot support any global steady-state $\boldsymbol{B}$ aligned electron flow within a simple standard two fluid model. For realistic parameter values, only non-stationary solutions can be expected. Albeit idealised, the model is not unphysical. It has been used often for simplified plasma studies and it can be realised in numerical simulations. Even though modifications of the model (as discussed in the following) give minor changes in the results, we can expect that the plasma conditions at large altitudes $\xi$ can be very sensitive to field-aligned electron drifts induced at small altitudes. In the following, we relax some of the simplifications made in Sect. 3.

\subsection{Ionisation and recombination}

We have so far ignored ionisation and recombination, which are important for ionospheric conditions. To include these effects in a steady-state model we generalise the quasi-neutral ion and electron continuity equations to become

$$
\frac{\mathrm{d} n u_{\mathrm{e}, \mathrm{i}}}{\mathrm{d} z}=\alpha-\beta n^{2},
$$


where $\alpha=\alpha(z)$ accounts for ionisation and $\beta$ is a recombination coefficient, which for simplicity we assume to be constant. Since $\alpha$ is proportional to the neutral density (usually approximated by an exponential variation), we have $\alpha(z) \rightarrow 0$ for $z \rightarrow \infty$. The continuity equations give

$u_{\mathrm{e}, \mathrm{i}}=\frac{1}{n} \int_{0}^{z}\left(\alpha-\beta n^{2}\right) \mathrm{d} z^{\prime}+u_{0, \mathrm{e}, \mathrm{i}} \frac{n_{0}}{n}$.

In particular we have

$$
\int_{0}^{z \rightarrow \infty}\left(\alpha-\beta n^{2}\right) \mathrm{d} z^{\prime} \rightarrow \mathrm{CR},
$$

with CR being a constant limit having the dimension of a flux density. The result (Eq. 13) will be used later on.

We now assume that ions as well as electrons lose all their momentum at recombination, and that this is the only momentum loss. In that case we can retain the electron and ion momentum equations in their original form to account for the dynamics between collisions. What is then missing is a frictional momentum loss due to elastic or inelastic electron and ion collisions with neutrals. To simplify the notation we retain $T_{\mathrm{i}}=0$ and $u_{0 \mathrm{i}}=0$ with little loss of generality. Within this model we obtain a closed equation in the form

$$
\begin{aligned}
& \frac{1}{2}\left(\int_{0}^{z}\left(\alpha-\beta n^{2}\right) \mathrm{d} z^{\prime}+u_{\mathrm{e} 0} n_{0}\right)^{2}= \\
& \quad-\frac{n^{2} M g}{m} z-\frac{T_{\mathrm{e}} n^{2}}{m} \ln \left(\frac{n}{n_{0}}\right)+\frac{1}{2} n^{2} u_{\mathrm{e} 0}^{2} .
\end{aligned}
$$

For large $z$ we can introduce the asymptotic limiting constant (Eq. 13) found for the left side to give

$$
(\mathrm{CR})^{2} \approx-\frac{n^{2} M g}{m} z-\frac{T_{\mathrm{e}} n^{2}}{m} \ln \left(\frac{n}{n_{0}}\right)+\frac{1}{2} n^{2} u_{\mathrm{e} 0}^{2} .
$$

In normalised units, where we let a constant $C^{2}$ represent the constant left side of Eq. (15), we find

$$
-C^{2} \approx \eta^{2}\left(\xi+\ln \eta-A^{2}\right),
$$

in general with $A \neq C$. Compared to the case $A=C$, see Eq. (5), we find that if $C<A$ then the singular point for the pressure force is moved to larger $\xi$, while $C>A$ implies that the singularity is found for smaller $\xi$. Ionisation and recombination changes the position of the singularity at $\xi_{\mathrm{c}}$ but does not remove it.

\subsection{Altitude varying parameters, including finite ion temperatures}

The basic model can be further modified and generalised to demonstrate that for a wide range of conditions the problem prohibits global steady-state conditions. The modifications included are finite ion pressures, $T_{\mathrm{i}} \neq 0$, and finite bulk ion flow velocities, $u_{\mathrm{i}} \neq 0$. The constant $A$ is generally small since we expect $u_{\mathrm{e}}<C_{\mathrm{S}}$ so $\xi_{\mathrm{c}}$ is generally a large altitude. Finite ion temperatures, in particular, will modify the simple constant electric field $E=M g / e$ that is found for the case with $T_{\mathrm{i}}=0$.

The divergence of a magnetic flux tube might be important, so we write the steady-state electron continuity equation as $u_{\mathrm{e}} n_{\mathrm{e}} F(z)=u_{\mathrm{e} 0} n_{0}$ where $F(z)$ accounts for an altitude varying magnetic flux tube cross-section. For a magnetic dipole, we use $F(z)=\left(z_{0}+z\right)^{3} / z_{0}^{3}$ where $z_{0}$ is the reference level taken to give $F(z=0)=1$. For ionospheric applications, we can take $z_{0}$ to be of the order of the Earth radius $R_{\mathrm{E}}$ with sufficient accuracy. The the flux-tube cross-section variation is the same for both ions and electrons.

For large $\xi_{\mathrm{c}}$ the assumption of a constant gravitational acceleration need not be fulfilled either so we replace the gravitational potential in Eq. (4) or (15) as $g z \rightarrow g z /\left(1+z / z_{0}\right)$, taking again the reference level for the gravity potential at $z=0$. Quasi neutrality, $n_{\mathrm{i}} \approx n_{\mathrm{e}} \equiv n$, is now an explicit approximation. The standard and by now classical model of Pannekoek (1922) and Rosseland (1924) is recovered for a static thermodynamic equilibrium with $T_{\mathrm{e}}=T_{\mathrm{i}}=$ const. More generally we have the equilibrium solution for constant electron and ion temperatures $n=n_{0} \exp \left(-(m+M) g /\left(T_{\mathrm{e}}+\right.\right.$ $\left.\left.T_{\mathrm{i}}\right)\right)$ with $e E=\left(M T_{\mathrm{e}}-m T_{\mathrm{i}}\right) g /\left(T_{\mathrm{e}}+T_{\mathrm{i}}\right) \approx M g T_{\mathrm{e}} /\left(T_{\mathrm{e}}+T_{\mathrm{i}}\right)$. The analysis includes the results of Sect. 3 as a special limit, but the general case does not allow a simple graphical interpretation as in Fig. 3.

To generalise the foregoing results from the simplified model, again we use the basic Eqs. (2) and (3) and assuming quasi-neutrality, $n_{\mathrm{e}} \approx n_{\mathrm{i}} \equiv n$, we find a generic form by adding Eqs. (2) and (3):

$$
\begin{aligned}
& \frac{1}{2} n \frac{\mathrm{d}\left(m u_{\mathrm{e}}^{2}+M u_{\mathrm{i}}^{2}\right)}{\mathrm{d} z}= \\
& \quad-\frac{\mathrm{d}\left(T_{\mathrm{e}}+T_{\mathrm{i}}\right) n}{\mathrm{~d} z}-M n \frac{g}{\left(1+z / z_{0}\right)^{2}} .
\end{aligned}
$$

Inclusion of momentum conserving electron-ion collisions in the original Eqs. (2) and (3) will not change the result (Eq. 17). The reference density $n_{0}$ does not appear in this formulation of the basic equation. Referring to the basic problem (see Fig. 2) we again assume that $u_{\mathrm{i}} \approx 0$ and denote the bulk electron velocity by $u$.

Here it turns out to be a simplification to express the equations in terms of the bulk electron flow velocity rather than the plasma density. Using the steady-state continuity equations in the general form $n u F=n_{0} u_{0}$, we therefore eliminate the plasma density to have

$\frac{m}{M} \frac{1}{F} \frac{\mathrm{d} u}{\mathrm{~d} z}=-\frac{\mathrm{d}}{\mathrm{d} z}\left(\frac{C_{\mathrm{s}}^{2}}{F u}\right)-\frac{1}{F u} \frac{g}{\left(1+z / z_{0}\right)^{2}}$.

We took $C_{\mathrm{s}}^{2} \equiv\left(T_{\mathrm{e}}+T_{\mathrm{i}}\right) / M$ and allow for an altitude variation of the temperatures, so that $C_{\mathrm{s}}^{2}=C_{\mathrm{s}}^{2}(z)$. With a little algebra 
we find that

$\frac{m}{M} \frac{\mathrm{d} u}{\mathrm{~d} z}=\frac{C_{\mathrm{s}}^{2}}{u^{2}} \frac{\mathrm{d} u}{\mathrm{~d} z}-\frac{F}{u} \frac{\mathrm{d}\left(C_{\mathrm{s}}^{2} / F\right)}{\mathrm{d} z}-\frac{1}{u} \frac{g}{\left(1+z / z_{0}\right)^{2}}$,

or

$$
\begin{aligned}
& \frac{1}{2}\left(\frac{m}{M}-\frac{C_{\mathrm{s}}^{2}}{u^{2}}\right) \frac{\mathrm{d} u^{2}}{\mathrm{~d} z}= \\
& \quad-\left(F \frac{\mathrm{d}\left(C_{\mathrm{s}}^{2} / F\right)}{\mathrm{d} z}+\frac{g}{\left(1+z / z_{0}\right)^{2}}\right),
\end{aligned}
$$

to be compared with Eq. (10). As for the solar wind problem (Parker, 1958; Cranmer, 2004; Parks, 2004) we find that a steadily increasing velocity $u$ with $\mathrm{d} u / \mathrm{d} z>0$ implies that an electron flow velocity that is slow at low altitudes, $u<$ $C_{\mathrm{s}} \sqrt{M / m} \equiv \sqrt{T_{\mathrm{e}} / m}$, and increasing, will make the left hand side of the expression change sign, so that at some point $z_{\mathrm{c}}$ we must have a change in sign of the right hand side as well, i.e.

$$
\left.F\left(z_{\mathrm{c}}\right) \frac{\mathrm{d}}{\mathrm{d} z}\left(\frac{C_{\mathrm{s}}^{2}(z)}{F(z)}\right)\right|_{z=z_{\mathrm{c}}}=-\frac{g}{\left(1+z_{\mathrm{c}} / z_{0}\right)^{2}},
$$

which defines the altitude where the electron flow velocity becomes super-thermal. For the special case with $C_{\mathrm{s}}$ constant and $F=\left(1+z / z_{0}\right)^{3}$ we have

$$
\left.\frac{1}{F} \frac{\mathrm{d} F}{\mathrm{~d} z}\right|_{z=z_{\mathrm{c}}}=\frac{3}{z_{0}} \frac{1}{1+z_{c} / z_{0}}=\frac{g / C_{\mathrm{s}}^{2}}{\left(1+z_{\mathrm{c}} / z_{0}\right)^{2}},
$$

giving $z_{\mathrm{c}}=z_{0}\left(\frac{1}{3} z_{0} g / C_{\mathrm{s}}^{2}-1\right)$. For realistic parameters we have $z_{0} \gg C_{\mathrm{s}}^{2} / g$ so this expression has solutions for $z>0$ in general, but at a distance $z_{\mathrm{c}}$ equaling many Earth radii. If $F=$ constant with a "flat Earth", we have no such solution. We do not know yet, however, whether this super-thermal flow can be reached by realistic injection velocities $u(0)=$ $u_{0}$. A solution $u^{2}=C_{\mathrm{s}}^{2} M / m=T_{\mathrm{e}} / m$ at some altitude where the right hand side of Eq. (20) differs from zero requires that $\mathrm{d} u^{2} / \mathrm{d} z$ becomes singular at this position.

To determine the position where the bracket-terms on the left side of Eq. (20) is zero, we first rewrite Eq. (20) as

$$
\begin{aligned}
\frac{m}{M} & \frac{\mathrm{d} u^{2}}{\mathrm{~d} z}-C_{\mathrm{s}}^{2} \frac{\mathrm{d} \ln u^{2}}{\mathrm{~d} z}= \\
& -2\left(F \frac{\mathrm{d}\left(C_{\mathrm{s}}^{2} / F\right)}{\mathrm{d} z}+\frac{g}{\left(1+z / z_{0}\right)^{2}}\right) .
\end{aligned}
$$

The right hand side is a known continuous function of $z$ and can in principle always be integrated to give

$$
\begin{aligned}
& K(z) \equiv \\
& -2 \int_{0}^{z}\left(F\left(z^{\prime}\right) \frac{\mathrm{d} C_{\mathrm{s}}^{2}\left(z^{\prime}\right) / F\left(z^{\prime}\right)}{\mathrm{d} z^{\prime}}+\frac{g}{\left(1+z^{\prime} / z_{0}\right)^{2}}\right) \mathrm{d} z^{\prime},
\end{aligned}
$$

so that

$$
\frac{m}{M} \frac{\mathrm{d} u^{2}}{\mathrm{~d} z}-C_{\mathrm{s}}^{2} \frac{\mathrm{d} \ln u^{2}}{\mathrm{~d} z}=\frac{\mathrm{d} K}{\mathrm{~d} z} .
$$

This equation can be solved numerically for given $C_{\mathrm{s}}=$ $C_{\mathrm{s}}(z)$ but gives difficulties for analytical results. Taking $C_{\mathrm{s}}$ to be constant we can, however, find

$$
K(z)=C_{\mathrm{s}}^{2} \ln F^{2}(z)+2 \frac{g z_{0}}{1+z / z_{0}},
$$

so that integration of Eq. (25) gives

$$
\begin{aligned}
& \frac{m}{M}\left(\frac{u}{C_{\mathrm{s}}}\right)^{2}-\ln \left(\frac{u}{C_{\mathrm{s}}}\right)^{2}= \\
& \quad \ln F^{2}(z)+2 \frac{z_{0} g / C_{\mathrm{s}}^{2}}{1+z / z_{0}}+\mathrm{CK} .
\end{aligned}
$$

The integration constant $\mathrm{CK}$ is determined by requiring $u(0)=u_{0}$. We can rewrite the previous expression as

$\ln \left(\frac{C_{\mathrm{s}}^{2}}{u^{2}} \exp \left(\frac{u^{2}}{C_{\mathrm{s}}^{2}} \frac{m}{M}\right)\right)=\ln F^{2}(z)+2 \frac{z_{0} g / C_{\mathrm{s}}^{2}}{1+z / z_{0}}+\mathrm{CK}$,

or

$$
\begin{aligned}
\frac{C_{s}^{2}}{u^{2}} & \exp \left(\frac{u^{2}}{C_{\mathrm{s}}^{2}} \frac{m}{M}\right) \\
& =\exp \left(\ln F^{2}(z)+2 \frac{z_{0} g / C_{\mathrm{s}}^{2}}{1+z / z_{0}}+\mathrm{CK}\right) \\
& =F^{2}(z) \exp \left(\frac{2 z_{0} g / C_{\mathrm{s}}^{2}}{1+z / z_{0}}\right) C_{1},
\end{aligned}
$$

introducing a new integration constant $C_{1}$ to be determined. With $F(0)=1$ we find

$C_{1}=\frac{C_{\mathrm{s}}^{2}}{u_{0}^{2}} \exp \left(\frac{u_{0}^{2}}{C_{\mathrm{s}}^{2}} \frac{m}{M}\right) \exp \left(-2 z_{0} g / C_{\mathrm{s}}^{2}\right)$.

Since $z_{0} g / C_{\mathrm{s}}^{2} \gg 1$ and generally $u_{0} \leq C_{\mathrm{s}}$ we expect $C_{1} \ll$ 1. We can solve for $u^{2} / C_{\mathrm{s}}^{2}$ and find in terms of Lambert's $W$ function

$$
\begin{aligned}
& \left(\frac{u}{C_{\mathrm{s}}}\right)^{2}= \\
& \quad-\frac{M}{m} W_{0}\left(-\frac{m / M}{F^{2}(z) C_{1}} \exp \left(-2 \frac{z_{0} g / C_{\mathrm{s}}^{2}}{1+z / z_{0}}\right)\right),
\end{aligned}
$$

which is a physically acceptable global solution when the right hand side is real and positive for all $z$. The corresponding expression for the normalised plasma density $\eta$ becomes

$$
\eta^{2}=-\frac{(m / M)\left(u_{0} / C_{\mathrm{s}}\right)^{2}}{W_{0}\left(-\frac{m / M}{F^{2}(z) C_{1}} \exp \left(-2 \frac{z_{0} g / C_{\mathrm{s}}^{2}}{1+z / z_{0}}\right)\right)} .
$$

Numerical solutions of Eq. (20) with altitude varying sound speeds are shown in Fig. 5, where we use a model for the altitude variation in the form $C_{\mathrm{s}}(z)=C_{\mathrm{s} 0}(1+$ 


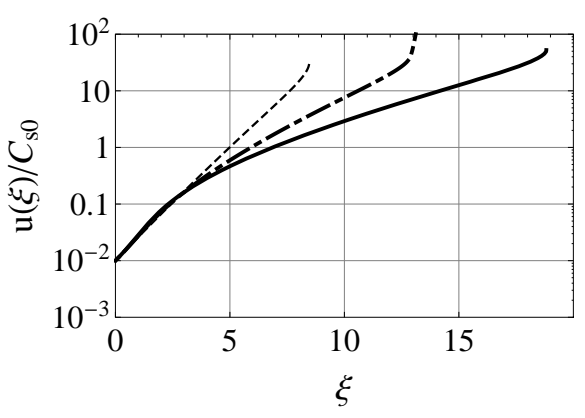

Figure 5. Numerical results for the electron fluid velocity with altitude varying sound speeds. We use the sound speed $C_{\mathrm{s} 0}$ at $z>0$ for normalisation. The heavy line has $A_{C}=2$; the dashed heavy line $A_{C}=1$. A thin dashed line gives the Lambert $W$ solution with constant $C_{\mathrm{S}}$ for reference, i.e. $A_{C}=0$. In all cases we have $u_{0}=0.01 C_{\mathrm{s} 0}$.

$\left.A_{C}(2 \arctan (z) / \pi)^{4}\right)$ with $A_{C}$ being a constant. The numerical solutions are inaccurate at the singularity, while the thin dashed line for $C_{\mathrm{s}}=$ constant uses the exact analytical result Eq. (29). The singularity in the velocity variation is delayed when the sound speed increases with altitude. The effect can qualitatively be accounted for by taking an enhanced average electron temperature instead of $T_{0}$. The restriction in using a constant sound speed is thus not significant. In Fig. 6 we show for completeness the variation of the normalised density with normalised altitude for the three cases shown in Fig. 5. The evolution of a steep vertical gradient can be noted. The pressure gradient becomes singular at this position.

Inspection of Eq. (17) indicates that the ion temperature merely adds to the electron temperature, and is consequently of minor importance. It is found, however, that $T_{\mathrm{i}} \neq 0$ changes the electric field from its constant value $E=M g / e$ obtained with $T_{\mathrm{i}}=0$ and $u_{\mathrm{i} 0}=0$ to $E=M g / e+$ $n^{-1} \mathrm{~d}\left(T_{\mathrm{i}} n\right) / \mathrm{d} z$ with $T_{\mathrm{i}}$ being a prescribed function of $z$, possibly a constant, while $n=n(z)=n_{0} u_{0} / u(z)$ is determined analytically when $u(z)$ is determined as shown in this section.

\subsection{Electron flow velocity solutions}

Some insight into the electron flow solution $u=u(z)$ can be found by rewriting Eq. (27) in dimensionless form by introducing $\vartheta \equiv\left(u / C_{\mathrm{s}}\right) \sqrt{m / M}$ and $\xi=z / L$ giving

$$
\begin{aligned}
& \frac{\exp \left(\vartheta^{2}\right)}{\vartheta^{2}}=\frac{\exp \left(\vartheta_{0}^{2}\right)}{\vartheta_{0}^{2}} \exp \left(-2 z_{0} / L\right) \\
& \times\left(1+\xi L / z_{0}\right)^{6} \exp \left(2 \frac{z_{0} / L}{1+\xi L / z_{0}}\right) \\
& \equiv \frac{\exp \left(\vartheta_{0}^{2}\right)}{\vartheta_{0}^{2}} Z_{0}(\xi) .
\end{aligned}
$$

The left side is a function of $\vartheta$ solely, while the right hand side is a function of $\xi$ only, with parameters being $M / \mathrm{m}$,

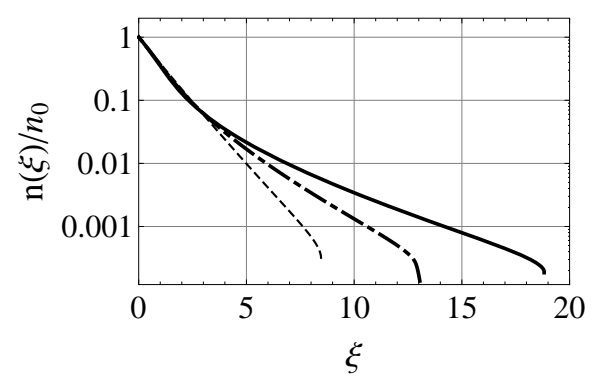

Figure 6. Variation of normalised density with normalised altitude for the three cases shown in Fig. 5.

$L / z_{0}$ and the normalised injection velocity $\vartheta_{0}$. The two former parameters are given by the plasma conditions. In particular we have $z_{0}$ equaling the Earth radius + the distance to the ionospheric $\mathrm{E}$ region, so that $z_{0} \approx R_{\mathrm{E}}$ where $R_{\mathrm{E}} \approx 6.4 \times 10^{6} \mathrm{~m}$ is the Earth radius, implying $L / z_{0} \ll 1$. For reference we show in Fig. 7 the left and right hand sides of Eq. (31), where we omit the multiplier $\exp \left(\vartheta_{0}^{2}\right) / \vartheta_{0}^{2}$ from the right hand side. Both expressions exist (are single valued) and are real for all values of the respective variables. It is clear that Eq. (31) will always have solutions, but for a physically acceptable solution we require it to exist and be real-valued for all altitudes $z$, i.e. all $\xi$. The left side has one and only one minimum at $\vartheta=1$ with functional value $\exp (1)$. The right hand side has also one and only one minimum value at $\xi=\frac{1}{3}\left(2-3 L / z_{0}\right) /\left(L / z_{0}\right)^{2}$ with functional value $(2 \exp (1) / 3)^{3} \exp \left(-2 L / z_{0}\right)\left(z_{0} / L\right)^{3} \exp \left(\vartheta_{0}^{2}\right) / \vartheta_{0}^{2}$. In order to have physically acceptable solutions we consequently require that the minimum value of the right hand side is equal to or larger than $\exp (1)$, otherwise there will be a $\xi$-interval without real solutions. This criterion gives the inequality

$$
\left(\frac{2 \exp (1)}{3}\right)^{3}\left(\frac{z_{0}}{L}\right)^{3} \exp \left(-2 \frac{z_{0}}{L}\right) \frac{\exp \left(\vartheta_{0}^{2}\right)}{\vartheta_{0}^{2}} \geq \exp (1),
$$

giving

$\frac{\exp \left(\vartheta_{0}^{2}\right)}{\vartheta_{0}^{2}} \geq\left(\frac{3}{2}\right)^{3}\left(\frac{L}{z_{0}}\right)^{3} \exp \left(2\left(\frac{z_{0}}{L}-1\right)\right)$.

The right hand side of the inequality Eq. (33) is illustrated in Fig. 8 for varying $L / z_{0}$. Typical values of $L$ in the ionosphere are $50-100 \mathrm{~km}$ and $z_{0} \approx R_{\mathrm{E}}$. Since $L / z_{0} \ll 1$ for conditions relevant to the Earth's ionosphere, we might conclude that it will never be possible to find a physically acceptable global solution for Eq. (33) and thereby a steady-state electron flow under the conditions described in Sect. 2 with $u_{\mathrm{e} 0}<C_{\mathrm{s}}$, corresponding to $\vartheta_{0}<\sqrt{m / M}$. Our conclusion is that only nonsteady flows should be expected in response to an induced electron flow, as described in Sect. 2, with reference to Fig. 2. Conditions for the existence of a steady electron outflow requires ionospheric temperatures much larger than those observed i.e. requiring much larger values of $L / R_{\mathrm{E}}$. 

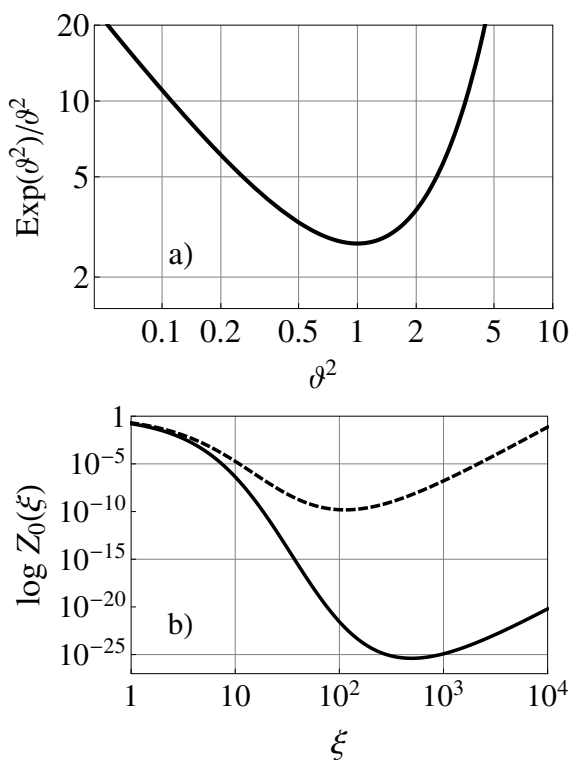

Figure 7. Illustration of the left and right hand sides of Eq. (31). In (a) we have the left side as a function of $\vartheta^{2}$ and in (b) we show $Z_{0}(\xi) \equiv \exp \left(-2 z_{0} / L\right)\left(1+\xi L / z_{0}\right)^{6} \exp \left(2\left(z_{0} / L\right) /(1+\right.$ $\left.\left.\xi L / z_{0}\right)\right)$. Note the different representations on the axes. In (b) we have the full line for $L / z_{0}=0.025$ and the dashed line for $L / z_{0}=0.05$.

The analysis of the present communication emphasises electron flows induced by a current injection mechanism as in Fig. 2, but we note the results can readily be generalised to account for ion flows as well.

\section{Conclusions}

The results presented here are as far as we could come analytically. Simple collisional friction terms for electrons and ions have been studied numerically, without causing changes in our conclusions. The results in this case also depend critically on small variable terms describing conditions in the lower part of the ionosphere. Also in this case we will have a large temporal variability of plasma conditions at large altitudes that need not be associated with local plasma instabilities. The lower parts of the ionosphere, the E region in particular, are often unstable with respect to excitation of low frequency, long wavelength perturbations that has been studied in great detail (Kelley, 1989), and these disturbances will cause fluctuations in $\xi_{\mathrm{c}}$.

The basic features of the solution (i.e. a singular point for the plasma pressure force) remain if ionisation and recombination are included, as well as variations of the cross-section of magnetic flux tubes, variation of the gravitational force with distance and altitude variations of electron and ion temperatures. The critical altitude $\xi_{\mathrm{c}}$ can be made large by taking small values of the injected electron flow velocity. A possibility for nontrivial steady-state current carrying solution is that

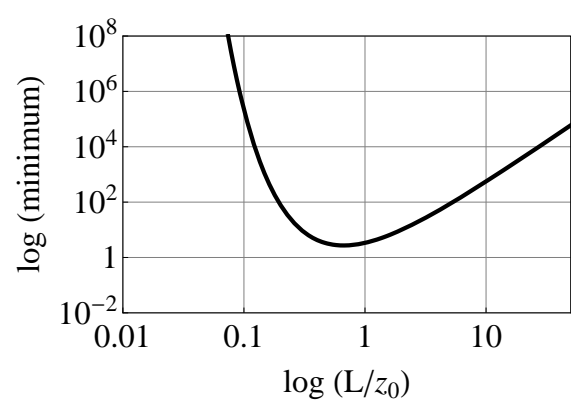

Figure 8. Illustration of the right hand side of the inequality Eq. (33) for varying $L / z_{0}$.

the altitude given by the critical altitude becomes too large to be of practical interest. It can thus be argued that if $\eta(\xi)$ exists (is real) up to values of $\xi_{\mathrm{c}}$ equaling many Earth radii, then the problem is not really relevant, but by this we implicitly impose a maximum value for $A^{2}$. Since $A^{2}$ is proportional to the imposed flow velocity in the boundary condition, this restriction is in reality the same as an electron velocity limit and thereby also a current density limitation. The location of a current closure region is most likely to be at some 10 Earthradii or more (Primdahl and Spangslev, 1983), implying that a steady-state well-behaved solution should have the pressure force singularity at a position larger than this. We estimate that an electron flow velocity $u_{0}$ should be less than $C_{\mathrm{S} 0}(M / m) \exp \left(-10 R_{\mathrm{E}} / L\right)$ to have the pressure singularity at an altitude of approximately $10 R_{\mathrm{E}}$ or more. For relevant cases this is a very small velocity. For most conditions we find the critical altitude to be smaller than $R_{\mathrm{E}}$.

We might speculate that a kinetic modelling, e.g. in the form of an electrostatic double layer, might be necessary to remedy the fluid singularity at $\xi_{\mathrm{c}}$. This conjecture could be resolved by numerical simulations.

A complete description of the battery mechanism discussed in Sect. 2 of this communication requires modelling the current closure, which for ionospheric conditions implies a study of the full inhomogeneous magnetic field configuration (Primdahl and Spangslev, 1977, 1983). This analysis is outside the scope of the present study.

The problem discussed in this work is related to the standard Parker model for the solar wind (Parker, 1958; Parks, 2004) and that problem has also found analytical results expressed by Lambert's $W$ function (Cranmer, 2004). Magnetic field-aligned density gradients can be generated also in laboratory plasmas (D’Angelo et al., 1976), and basic elements of the present analysis can be applied there as well. Although these and other problems are mathematically related to the topic of the present study, it should be emphasised that the physical conditions, the boundary conditions in particular, are different and of a different nature. A general framework for combining these problems might be found (Kruskal, 1965). 
Acknowledgements. Parts of this study were carried out while one of the authors (H. L. Pécseli) was visiting Tokyo University of Agriculture and Technology (TUAT). This author thanks S. Shinohara for organising this visit, thanking also students and staff at TUAT for their kind hospitality. The original figure used for obtaining the present Fig. 1 was provided by the late Klaus Rinnert for a different study.

Topical Editor S. Milan thanks the two anonymous referees for their help in evaluating this paper.

\section{References}

Arnoldy, R. L.: Auroral particle precipitation and Birkeland currents, Rev. Geophys., 12, 217-231, doi:10.1029/RG012i002p00217, 1974.

Boström, R.: A model for the Auroral electrojet, J. Geophys. Res., 69, 4983-4999, doi:10.1029/JZ069i023p04983, 1964.

Cranmer, S. R.: New views of the solar wind with the Lambert W function, Am. J. Phys., 72, 1397-1403, doi:10.1119/1.1775242, 2004.

D’Angelo, N., Michelsen, P., and Pécseli, H. L.: Ion-acoustic-waves in a density gradient, Z. Naturforsch. A, 31, 578-582, 1976.

de la Beaujardiere, O., Watermann, J., Newell, P., and Rich, F.: Relationship between Birkeland current regions, particle precipitation, and electric fields, J. Geophys. Res.-Space, 98, 7711-7720, doi:10.1029/92JA02005, 1993.

Dyrud, L., Krane, B., Oppenheim, M., Pécseli, H. L., Schlegel, K., Trulsen, J., and Wernik, A. W.: Low-frequency electrostatic waves in the ionospheric E-region: a comparison of rocket observations and numerical simulations, Ann. Geophys., 24, 29592979, doi:10.5194/angeo-24-2959-2006, 2006.

Garcia, O. E. and Pécseli, H. L.: Models for electrostatic drift waves with density variations along magnetic field lines, Geophys. Res. Lett., 40, 5565-5569, doi:10.1002/2013GL057802, 2013.

Gurevich, A. V.: Nonlinear Phenomena in the Ionosphere, Vol. 10 of Physics and Chemistry in Space, Springer, New York, 1978.

Kelley, M. C.: The Earth's Ionosphere, Plasma Physics and Electrodynamics, Vol. 43 of International Geophysics Series, Academic Press, San Diego, California, 1989.

Khotyaintsev, Y., Lizunov, G., and Stasiewicz, K.: Langmuir wave structures registered by FREJA: analysis and modeling, Adv. Space Res., 28, 1649-1654, doi:10.1016/S0273-1177(01)004859, 2001.

Kindel, J. M. and Kennel, C. F.: Topside current instabilities, J. Geophys. Res., 76, 3055-3078, doi:10.1029/JA076i013p03055, 1971.

Knight, S.: Parallel electric fields, Planet. Space Sci., 21, 741-750, doi:10.1016/0032-0633(73)90093-7, 1973.
Kruskal, M. D.: Asymtotology, in: Plasma Physics, edited by: Kadomtsev, B. B., Rosenbluth, M. N., and Thompson, W. B., 373-387, IAEA, Vienna, 1965.

Lyons, L. R., Evans, D. S., and Lundin, R.: An observed relation between magnetic field aligned electric fields and downward electron energy fluxes in the vicinity of auroral forms, J. Geophys. Res.-Space, 84, 457-461, doi:10.1029/JA084iA02p00457, 1979.

Ossakow, S. L. and Chaturvedi, P. K.: Current convective instability in the diffuse Aurora, Geophys. Res. Lett., 6, 332-334, doi:10.1029/GL006i004p00332, 1979.

Pannekoek, A.: Ionisation in stellar atmospheres, B. Astron. I. Netherlands, 1, 107-122, 1922.

Parker, E. N.: Dynamics of the interplanetary gas and magnetic fields, Astrophys. J., 128, 664-676, doi:10.1086/146579, 1958.

Parks, G. K.: Physics of Space Plasmas, an Introduction, 2nd Edn., Westview Press, Cambridge, Massachusetts, 2004.

Primdahl, F. and Spangslev, F.: Cross-polar cap horizontal E region currents related to magnetic disturbances and to measured electric fields, J. Geophys. Res., 82, 1137-1143, doi:10.1029/JA082i007p01137, 1977.

Primdahl, F. and Spangslev, F.: Does IMF By induce the cusp field-aligned currents?, Planet. Space Sci., 31, 363-367, doi:10.1016/0032-0633(83)90150-2, 1983.

Primdahl, F., Walker, J., Spangslev, F., Olesen, J., Fahleson, U., and Ungstrup, E.: Sunlit cleft and polar cap ionospheric currents determined from rocket-borne magnetic field, plasma, and electric field observations, J. Geophys. Res., 84, 6458-6470, doi:10.1029/JA084iA11p06458, 1979.

Primdahl, F., Marklund, G., and Sandahl, I.: Rocket observation of E-B-field correlations showing up- and down-going Poynting flux during an auroral breakup event, Planet. Space Sci., 35, 1287-1295, doi:10.1016/0032-0633(87)90114-0, 1987.

Prölss, G. W.: Physics of the Earth's Space Environment, an Introduction, Springer, Berlin, 2004.

Rosseland, S.: Electrical state of a star, Monthly Notices Royal Astron. Soc., 84, 720-729, doi:10.1093/mnras/84.9.720, 1924.

Smiddy, M., Kelley, M. C., Burke, W., Rich, F., Sagalyn, R., Shuman, B., Hays, R., and Lai, S.: Intense poleward-directed electric fields near the ionospheric projection of the plasmapause, Geophys. Res. Lett., 4, 543-546, doi:10.1029/GL004i011p00543, 1977.

Stubbe, P.: Frictional forces and collision frequencies between moving ion and neutral gases, J. Atmos. Terr. Phy., 30, 1965-1985, doi:10.1016/0021-9169(68)90004-4, 1968.

Valluri, S. R., Jeffrey, D. J., and Corless, R. M.: Some applications of the Lambert W function to physics, Can. J. Phys., 78, 823831, doi:10.1139/p00-065, 2000. 